\title{
THE
}

\section{Changes in selection pressure can facilitate hybridization during biological invasion in a Cuban lizard}

\author{
Dan G. Bock \\ Simon Baeckens \\ Jessica N. Pita-Aquino \\ University of Rhode Island \\ Zachary A. Chejanovski \\ University of Rhode Island \\ Sozos N. Michaeolides
}

Follow this and additional works at: https://digitalcommons.uri.edu/bio_facpubs
The University of Rhode Island Faculty have made this article openly available. Please let us know how Open Access to this research benefits you.

This is a pre-publication author manuscript of the final, published article.

Terms of Use

This article is made available under the terms and conditions applicable towards Open Access Policy Articles, as set forth in our Terms of Use.

\section{Citation/Publisher Attribution}

Bock, D. G., Baeckens, S., Pita-Aquino, J. N., Chejanovsku, Z. A., Michaelides, S. N., Muralidhar, P.,...Kolbe, J. J. (2021). Changes in selection pressure can facilitate hybridization during biological invasion in a Cuban lizard. PNAS, 118(42), e2108638118. https://doi.org/10.1073/pnas.2108638118

Available at: https://doi.org/10.1073/pnas.2108638118 


\section{Authors}

Dan G. Bock, Simon Baeckens, Jessica N. Pita-Aquino, Zachary A. Chejanovski, Sozos N. Michaeolides, Pavitra Muralidhar, Sungdae Park, Douglas B. Menke, Anthony J. Geneva, Jonathan B. Losos, and Jason J. Kolbe 
1 Title Changes in selection pressure can facilitate hybridization during biological

2 invasion in a Cuban lizard

4 Authors: Dan G. Bock ${ }^{\mathrm{a}, 1}$, Simon Baeckens ${ }^{\mathrm{b}}$, Jessica N. Pita-Aquino ${ }^{\mathrm{c}}$, Zachary A. Chejanovskic,

5 Sozos N. Michaelides ${ }^{\mathrm{c}, \mathrm{d}}$, Pavitra Muralidhare, Oriol Lapiedra ${ }^{\mathrm{f}}$, Sungdae Park ${ }^{\mathrm{g}}$, Douglas B.

6 Menkes, Anthony J. Geneva ${ }^{\mathrm{h}}$, Jonathan B. Losos ${ }^{\mathrm{a}, \mathrm{i}, 1,2}$, Jason J. Kolbe $\mathrm{e}^{\mathrm{c}, 2}$

$8 \quad{ }^{a}$ Department of Biology, Washington University in St. Louis, St. Louis, MO 63130

$9 \quad{ }^{b}$ Functional Morphology Lab, Department of Biology, University of Antwerp, B-2610 Wilrijk,

10 Belgium

$11{ }^{\mathrm{c}}$ Department of Biological Sciences, University of Rhode Island, Kingston, RI 02881

$12{ }^{\mathrm{d}}$ Department of Biology, Concordia University, Montreal, QC H4B 1R6, Canada

$13{ }^{\mathrm{e}}$ Center for Population Biology and Department of Evolution and Ecology, University of

14 California, Davis, CA 95616

$15{ }^{\mathrm{f}}$ CREAF Centre for Ecological Research and Applied Forestries, Cerdanyola del Valles,

16 Catalonia 08193, Spain

$17 \mathrm{~g}$ Department of Genetics, University of Georgia, Athens, GA 30602

$18{ }^{\mathrm{h}}$ Department of Biology and Center for Computational and Integrative Biology, Rutgers

19 University, Camden, NJ, 08102

20 i Living Earth Collaborative, Washington University in St. Louis, St. Louis, MO 63130

21 Competing interest statement: The authors declare no competing interests.

221 correspondence to: dan.g.bock@gmail.com (to D.G.B) or losos@wustl.edu (to J.B.L)

$23 \quad 2$ These authors contributed equally to this work 


\section{Abstract}

25 Hybridization is among the evolutionary mechanisms most frequently hypothesized to drive the

26 success of invasive species, in part because hybrids are common in invasive populations. One

27 explanation for this pattern is that biological invasions coincide with a change in selection

28 pressures that limit hybridization in the native range. To investigate this possibility, we studied

29 the introduction of the brown anole (Anolis sagrei) in the southeastern United States. We find

30 that native populations are highly genetically structured. In contrast, all invasive populations

31 show evidence of hybridization among native-range lineages. Temporal sampling in the invasive

32 range spanning 15 years showed that invasive genetic structure has stabilized, indicating that

33 large-scale contemporary gene flow is limited among invasive populations and that hybrid

34 ancestry is maintained. Additionally, our results are consistent with hybrid persistence in

35 invasive populations resulting from changes in natural selection that occurred during invasion.

36 Specifically, we identify a large-effect X chromosome locus associated with variation in limb

37 length, a well-known adaptive trait in anoles, and show that this locus is often under selection in

38 the native range, but rarely so in the invasive range. Moreover, we find that the effect size of

39 alleles at this locus on limb length is much reduced in hybrids among divergent lineages,

40 consistent with epistatic interactions. Thus, in the native range, epistasis manifested in hybrids

41 can strengthen extrinsic post-mating isolation. Together, our findings show how a change in

42 natural selection can contribute to an increase in hybridization in invasive populations. 


\section{Significance}

44 Hybridization is common in invasive species and can be important for their success. The

45 connection between hybridization and bioinvasions could result in part because of a disruption in

46 the selection pressures that limit hybridization in the native range. We demonstrate that, in the

47 lizard Anolis sagrei, hybridization is rare in native populations, which show frequent evidence of

48 natural selection at a large effect X chromosome locus. Conversely, little selection at this locus

49 was detected in invasive populations, which do not experience large-scale contemporary gene

50 flow, but instead maintain a mosaic of hybrid ancestries formed during invasive range

51 colonization. Ecological changes during biological invasions can affect hybridization frequency

52 and stability, which can in turn drive the success of invasive taxa. 


\section{Introduction}

54 Evolutionary change can affect the success of invasive species. This possibility has been

55 considered by biologists for more than half a century (1). Recent decades, however, have seen a

56 remarkable growth in research on this topic (2-5), simultaneous with the greater emphasis on

57 contemporary evolution driving ecological phenomena $(6,7)$. It is now widely accepted that

58 substantial evolution can occur over a few generations, well within the timeframe in which the

59 establishment and spread stages of biological invasions play out $(6,7)$.

60

61 Among evolutionary factors that can facilitate invasions, hybridization is noteworthy for several

62 reasons (8-10). For one, hybridization between species or among lineages within species has

63 repeatedly been observed in genetic surveys of invasive taxa. As a result, invasive populations

64 often show levels of genetic diversity equal to or larger than those in native populations (11-14).

65 Also, meta-analyses have shown that invasive hybrids are frequently larger and more fecund than

66 their parents (15). Moreover, genetically, hybridization can contribute to invasive spread through

67 hybrid vigor or adaptive introgression $(12,13,16)$.

68

69 Nonetheless, studies directly connecting hybridization to invasive spread are still in the minority.

70 As a result, we do not know how often hybridization is a true driver of invasion success, or a

71 consequence of propagule pressure and repeated introductions of allopatric lineages (10).

72 Further, why hybridization is less common in the native range than in the invasive range of some

73 species is unclear, particularly when opportunities for human-mediated inter-population dispersal

74 and contemporary physical barriers to natural dispersal do not clearly differ between ranges (e.g., 75 refs. 14 and 17). 
77 Changes in natural selection that occur during biological invasions may provide part of the

78 answer for why hybrids are more common in invasive populations, especially when biological

79 invasions occur in disturbed habitats or in habitats that are ecologically novel from the

80 perspective of the invader. In a landmark paper on hybridization and invasiveness, Ellstrand \&

81 Schierenbeck (9) discussed 28 species for which hybridization preceded invasive spread. All of

82 these were found to occur in habitats characterized by disturbance, indicating that the change of

83 selection pressures experienced by parental lineages or the opening of new niches to which

84 hybrids are better adapted might facilitate hybridization in invasive species (9). Originally

85 proposed by Anderson (18), the possibility that unstable, rapidly changing or novel habitats

86 promote hybridization is now well documented in non-invasive species (19). Particularly

87 compelling examples come from long-term field studies that show a lack of hybridization before

88 the environment changes (20) or a return to non-hybrid status once the ancestral environment is

89 restored (21). In a similar way, hybrids may more easily form or persist in invasive populations

90 when biological invasions coincide with a temporary or permanent change in patterns of

91 adaptation that evolved in the native range $(22,23)$.

92

93 To investigate whether changes in selection may facilitate hybridization during biological

94 invasions, we studied the Cuban brown anole (Anolis sagrei), an excellent organism for such

95 research for multiple reasons. First, it is one of the best-known examples of hybridization

96 occurring during biological invasion. Previous studies indicated that most invasive populations in

97 Florida, in the southeastern United States, derive from admixture between divergent native-range

98 lineages $(11,24)$. Second, A. sagrei is a highly successful invader. Since the mid- to late-1800's, 
99 when the first populations were established in Florida, the species has colonized the entire 100 peninsula and expanded to the north and west (11). From there, it has since seeded secondary

101 invasions globally (11). Third, niche structure, natural selection and local adaptation are

102 exceptionally well-studied in anoles, thanks to decades of observational and experimental work

103 (25). A number of these studies focused on natural and experimental populations of A. sagrei in 104 its native range in the Caribbean, predominantly on islands in the Bahamas (e.g., refs. 26-33). As 105 such, an important baseline regarding phenotypic and environmental components of native range 106 local adaptation is available in this system.

\section{Results and Discussion}

\section{Genetic structure and genetic diversity in native and invasive A. sagrei}

110 We first aimed to understand how genetic variation is partitioned among native and invasive 111 populations. For the native range, we included 10 populations, nine of which are representative

112 of lineages known to have seeded the Florida invasion (11). For the invasive range, we included 11334 populations predominantly from Florida (SI Appendix, Fig. S1 and Dataset S1). We obtained 114 genome-wide SNPs for all lizards $(N=824)$ via double-digest RAD-seq reduced representation 115 sequencing (SI Appendix, Methods). Principal component analyses (PCA), phylogenetics, and 116 Bayesian clustering all pointed towards strong genetic structure in the native range producing six 117 main lineages, with limited evidence of among-lineage genetic exchange (Fig. 1A, B; SI 118 Appendix, Figs. S2-S4). Specifically, only one of the 134 genotypes was clearly a hybrid (Fig.

119 1A). Further genetic structure was apparent within clades, albeit to a lesser extent (Fig. 1A, 120 inset). 
122 Previous studies have indicated that geographical distance is an important determinant of genetic 123 structure for $A$. sagrei in Cuba $(34,35)$. Our results reinforce these findings by providing

124 evidence of strong isolation by distance (IBD; Mantel's $r=0.88, P=5 \times 10^{-4}$; SI Appendix, Fig.

125 S6A). However, contrary to expectations under true IBD, genetic distances did not increase

126 uniformly with geographic distance. Rather, moderate within-clade genetic structure was

127 complemented by much stronger among-clade genetic subdivisions (SI Appendix, Fig. S6A).

128 Similar hierarchical genetic structure has been shown to lead to significant IBD (36). Strong

129 among-clade genetic fragmentation has been found in the native range of other anole species 130 that, like $A$. sagrei, have a broad and continuous distribution (discussed in ref. 25). The fact that

131 some of the genetic breaks, including those described in Cuban A. sagrei, overlap in different

132 species while also corresponding to inferred past geological barriers (35) suggests that they

133 resulted from divergence in allopatry, likely during periods of partial island submergence (35, $13437)$.

136 The mechanisms that allow these genetic subdivisions to be maintained in the absence of 137 contemporary geographical barriers are, however, unknown. This genetic differentiation is 138 particularly striking given that $A$. sagrei clades have geographically abutting distributions in 139 Cuba (35). One possibility is that local adaptation and resulting ecological selection against 140 immigrants and hybrids is involved. Such ecological structuring of genetic variation across the 141 landscape is suggested by results from previous studies that indicated ecology has an important 142 role in shaping spatial genetic divergence in anoles, albeit to a lesser extent as compared to 143 geographical distance (34). We revisit the contribution of local adaptation below. 
145 Compared to the native range, population structure in the invasive range was markedly different.

146 There was no clear grouping of genotypes in PCA space (Fig. 1A), and all populations showed

147 evidence of admixture, deriving ancestry from more than one native Cuban lineage (Fig. 1B; SI

148 Appendix, Figs. S3 and S5). Also, within-population estimates of relatedness were more similar

149 to among-population estimates of relatedness in the invasive range than in the native range,

150 indicating that ancestral population structure has collapsed during invasion (SI Appendix, Fig.

151 S7). Lastly, the strength of IBD was much reduced relative to the native range (Mantel's $r=$

$1520.28, P=0.004$; SI Appendix, Fig. S6B), as expected given that dispersal of invasive $A$. sagrei

153 occurred with the contribution of human-mediated long-distance transport.

155 Shifts in genetic structure between the native and invasive ranges were accompanied by

156 important changes in genetic variation. Genome-wide heterozygosity, which we used as a proxy

157 for neutral genetic variation, was almost completely non-overlapping between the two ranges,

158 with invasive populations significantly more diverse (two-sided Wilcoxon rank-sum test, $P=$

$1594.719 \times 10^{-9}$; Fig. 1C; SI Appendix, Fig. S8A). This pattern was reversed for SNPs predicted to

160 be detrimental - those that produce premature stop codons, frameshift mutations, or the loss of

161 start codons (SI Appendix, Methods). Invasive populations showed a lower proportion of these

162 putatively deleterious mutations as compared to native populations (two-sided Wilcoxon rank-

163 sum test, $P=0.015$; Fig. 1C; SI Appendix, Fig. S8B), indicating that the purging of genetic load

164 occurred more readily in the invasive range. This could have happened if invasion was

165 accompanied by a change in the fitness landscape, such that weakly deleterious alleles in native

166 populations are more readily visible to selection in the new environment (38). As well, if

167 recessive, even strongly deleterious alleles would have persisted at low frequency in native 
168

169

170

\section{Invasive genetic structure has stabilized to a mosaic of hybrid ancestries}

172 The observation of widespread hybridization in invasive $A$. sagrei is notable given that hybrids

173 among divergent lineages are rare in the native range. To investigate whether this is due to the

174 more recent history of $A$. sagrei in Florida, such that removal of hybrids in these populations is 175 ongoing, we resampled a subset of invasive populations fifteen years after they were first 176 sampled. We included SNPs from six Florida populations $(N=172)$ that we surveyed in both 1772003 and 2018 (SI Appendix, Fig. S9).

178

179 180 181

182

populations. These rare alleles should have been preferentially lost during genetic bottlenecks that occurred when invasive populations were seeded. sampled. We included SNPs from six Florida populations $(N=172)$ that we surveyed in both

We found that hybrid ancestry is not eliminated in Florida, and that genetic differences among populations resulting from independent introduction and hybridization events are maintained over at least 15 years. First, genetic structure inferred using PCA did not change between 2003 and 2018 (SI Appendix, Fig. S10A). Second, while populations differed in the proportion of Western Cuba ancestry $\left(F_{5,165}=217.4 ; P<2 \times 10^{-16}\right.$; SI Appendix, Fig. S10B), reflecting the mosaic of hybrid origins that occur across Florida (Fig. 1B; see also ref. 11), each population maintained similar proportions over the 15 -year period $\left(F_{1,165}=0.207 ; P=0.649 ;\right.$ SI Appendix, Fig. S10B). Third, there was no effect of sampling time on the index of admixture $\left(F_{1,165}=\right.$ 0.972; $P=0.325 ;$ SI Appendix, Fig. S10C), which takes into account the contribution of each native-range lineage to the ancestry of hybrids (12). 
190 The only metric that did change was heterozygosity, which decreased significantly over the 15

191 years across all populations $\left(F_{1,165}=50.72 ; P=3.12 \times 10^{-11}\right.$; SI Appendix, Fig. S11A $)$. Two lines

192 of evidence suggest that this drop in heterozygosity is not a result of ongoing purging of common

193 intrinsic incompatibilities, but is instead driven by the loss of rare alleles, likely due to genetic

194 drift. First, heterozygosity decreased irrespective of whether hybrids originated from closely

195 related or distantly related lineages (SI Appendix, Fig. S11B). This is inconsistent with selection

196 against intrinsic incompatibilities, which should be more common in hybrids among divergent

197 lineages (39). Second, polymorphism lost in the 2018 samples involved alleles that were already

198 rare in 2003 (SI Appendix, Fig. S11C), consistent with genetic drift. We note, however, that

199 these results do not exclude the possibility that any intrinsic incompatibilities have been purged

200 by selection prior to 2003 , the first timepoint represented in our sampling.

202 The finding that genetic differences established among Florida populations by 2003 remain 203 unchanged by 2018 argues against large-scale ongoing gene flow in the invasive range. Such 204 gene flow would have been expected to shift population genetic structure over the span of 15 205 years. Rather, a more plausible interpretation for hybrid ancestry in Florida populations is that 206 distinct hybridization events took place during the evolutionary history of invasive A. sagrei.

207 These hybridization events would have occurred when divergent lineages met after repeated 208 introductions from Cuba and following progressive range expansion from these points of initial 209 introduction.

211 An equally important finding is that hybrid ancestry is not only common, but it is also stable in 212 the invasive range. This contrasts with observations in the native range where hybrids are much 
213 rarer, particularly among lineages that are representative of major phylogenetic splits in $A$.

214 sagrei, and even when these lineages have geographically abutting distributions (e.g., the Central

215 Cuba and East-Central Cuba lineages; SI Appendix, Fig. S2). Limited genetic exchange among

216 these divergent lineages in the native range is supported by our data as well as a previous study

217 that surveyed more Cuban populations (35). Differences in hybridization frequency between the

218 native and invasive ranges suggest that at least some gene flow barriers in the native range are

219 conditional on environment (i.e., they are extrinsic). If barriers to gene flow were predominantly

220 unconditional (i.e., represented by intrinsic genetic incompatibilities), we would have expected

221 to find that hybridization is equally rare in both ranges or that hybrid ancestry present in invasive

222 populations is progressively eliminated.

223

224 Genome scans are consistent with changes in natural selection

225 To understand differences in hybridization frequency between ranges, we focused on local

226 adaptation, which can limit gene flow between populations that inhabit different environments

$227(40,41)$. To this end, we contrasted the genomic signature of selection for the native and

228 invasive ranges. In the native range, population differentiation was strongest on the $\mathrm{X}$

229 chromosome, where fixed differences $\left(F_{\mathrm{ST}}=1\right)$ occurred even among closely related

230 populations, for which median genome-wide $F_{\mathrm{ST}}$ was over 26 times lower than on the $\mathrm{X}$

231 chromosome $\left(F_{\mathrm{ST}}=0.038\right.$; Fig. 2A; SI Appendix, Fig. S12). High $\mathrm{X}$ chromosome $F_{\mathrm{ST}}$ windows

232 were concentrated in a region of $18 \mathrm{Mbp}$ in size (chromosome 7 coordinates $70 \mathrm{Mbp}-88 \mathrm{Mbp}$;

233 Fig. 2B). While representing $16 \%$ of the $\mathrm{X}$ chromosome length, this locus accounted for $47 \%$ of

234 all X chromosome outliers and $62 \%$ of extreme X-chromosome outliers. 
236 Compared to the native range, genome-wide $F_{\mathrm{ST}}$ in the invasive range was only a third as high

237 (Fig. 2A) and there were no fixed differences among sampling sites (all windowed $F_{\text {ST }}<1$; SI

238 Appendix, Fig. S13). Even so, the genomic landscape was similar to the one we observed in the

239 native range, with the $\mathrm{X}$ chromosome contributing more to population differentiation (Fig. 2A;

240 SI Appendix, Fig. S13). Compared to the native range, however, the largest X chromosome $F_{\text {ST }}$

241 peaks resulted from comparisons among divergent lineages (SI Appendix, Fig. S13), which we

242 could infer for invasive hybrids at this chromosomal region due to reduced recombination (SI

243 Appendix, Methods). This indicates that introduction history, which can create sharp breaks in

244 allele frequencies among populations (42), likely has an important role in elevating invasive

245 range $F_{\mathrm{ST}}$ at this genomic region. Specifically, because this locus is frequently differentiated

246 among native range populations, any two invasive populations with $\mathrm{X}$ chromosome haplotypes

247 of different origins will also show high $\mathrm{X}$ chromosome $F_{\mathrm{ST}}$. Lastly, outliers were less

248 concentrated in the divergent $\mathrm{X}$ chromosome region identified from native populations (Fig. 2B).

249 In invasive populations, this locus contained $35 \%$ of all $\mathrm{X}$ chromosome outliers and $43 \%$ of

250 extreme X chromosome outliers. After accounting for introduction history by excluding

251 comparisons made among divergent X chromosome lineages, these estimates dropped to $19 \%$

252 and $25 \%$, respectively.

253

254 We next asked whether the same outliers have repeatedly contributed to differentiation among

255 independent population pairs from within either the native or the invasive ranges. Non-parallel

$256 F_{\text {ST }}$ windows accounted for the majority of outliers (85.4\%-89.2\%; SI Appendix, Table S1).

257 While representing a smaller fraction of the genome, repeated outliers were more common than

258 expected by chance, for both ranges $(P<0.001$ all permutation tests; SI Appendix, Fig. S14). 
259 Also, relative to null expectations, there were more repeated outliers in the invasive range than in

260 the native range (SI Appendix, Fig. S14). Repeated $F_{\mathrm{ST}}$ differentiation in both ranges is

261 consistent with divergence under natural selection. In the invasive range, higher repeatability can

262 be the result of the increased linkage disequilibrium that characterizes these populations (SI

263 Appendix, Fig. S15), thereby enhancing non-independence of adjacent genomic windows. While

264 the genome-wide proportion of repeated outliers was larger in invasive populations, this pattern

265 was reversed at the X chromosome locus, which contributed proportionately more to parallel

266 differentiation in the native range (SI Appendix, Fig. S16). This further underscores that the

267 genetic basis of population differentiation is different between the two ranges, with the $\mathrm{X}$

268 chromosome locus having a larger contribution in the native range.

270 In support of $F_{\mathrm{ST}}$ results, we found that Tajima's $D$ is often reduced at the same $\mathrm{X}$ chromosome

271 locus in native populations, but rarely so in invasive populations (Fig. 2C; SI Appendix, Figs

272 S17-S18). Reduced Tajima's D indicates an excess of low-frequency variants, as expected after

273 positive or negative selection (43). Among these possibilities, positive selection was likely

274 involved, as indicated by Fay and Wu's $H$ test (Fig. 2D). This metric quantifies the excess of

275 derived (i.e., non-ancestral) alleles, which is expected under positive selection. We found that $H$

276 was lower in the native range than in the invasive range at the $\mathrm{X}$ chromosome divergent locus

277 (one-sided Wilcoxon rank-sum test, $P=1.26 \times 10^{-6}$ ). Also, $H$ estimates at this genomic region

278 were lower than background neutral values only for native populations (Fig. 2D), consistent with

279 positive selection in the native range only. Background neutral $H$ values were similar between

280 the native and invasive ranges (one-sided Wilcoxon rank-sum test, $P=1$ ), indicating that

281 admixture or demography, which should impact genetic variation more broadly rather than a 
282 single locus, are unlikely to be a major source of bias in our results. Moreover, positive selection

283 should have been easy to identify using Fay and Wu's $H$ in invasive populations, because this

284 metric has more power in admixed than in non-admixed populations (44). Lastly, we note that

285 while analyses presented above focused on the most recent (i.e., 2018) samples from the invasive

286 range, we also contrasted $H$ between the 2003 and 2018 timepoints, for the six invasive

287 populations with temporal data. These analyses provided no evidence of change in $H$ values in

288 the invasive range, at least over the span of the 15 years covered in our sampling (Wilcoxon

289 rank-sum tests, all $P>0.05)$.

290

291 Compared to metrics discussed above, the signature of selection persists in nucleotide diversity

$292(\pi)$ and absolute differentiation $\left(D_{\mathrm{xy}}\right)$ for more generations $(45,46)$. Therefore, $\pi$ and $D_{\mathrm{xy}}$ can be

293 informative with regards to whether selection also occurred in the common ancestor of

294 populations under investigation. Consistent with a "selective sweep before selective population

295 differentiation" model $(47,48)$, we found that both metrics tend to be reduced at the same X-

296 linked locus relative to the rest of the chromosome in both ranges (Fig. 2E; SI Appendix, Figs.

297 S19-S22). These findings highlighting an important role of this locus to local adaptation in the

298 native range, and at multiple points throughout the evolutionary history of $A$. sagrei.

300 In summary, we find that the genomic signature of selection is different between the two ranges.

301 In the native range, an $\mathrm{X}$ chromosome locus frequently retains a signature consistent with

302 positive selection. By contrast, the same genomic region is evolving neutrally in most invasive

303 populations. Two non-mutually exclusive explanations can account for these results. First, if

304 evolution in both ranges is driven by similar selective forces, changes in the genomic target of 


\section{5}

selection could have occurred in invasive populations, following hybridization among divergent lineages and introgression of adaptive alleles at other loci in the genome. We consider this less likely, given that the $\mathrm{X}$ chromosome locus is part of the genetic architecture of local adaptation in most native-range lineages that contributed ancestry to invasive populations. Also, relative to native populations, most invasive populations showed limited evidence of selection at the $\mathrm{X}$ chromosome locus, despite varying in ancestry and extent of hybridization. The second explanation is that temporary or permanent changes in selection pressure occurred during biological invasion in A. sagrei.

Changes in selection pressure can contribute to differences in hybridization frequency that we observe between ranges. For example, to the extent that dispersal in the native range occurs between populations that are adapted to different environments, natural selection is expected to limit within or among clade gene flow via both pre-mating and post-mating mechanisms (40, 41), thereby enhancing population genetic structure. Pre-mating isolation occurs when selection removes maladapted immigrant genotypes before these can produce hybrid offspring (40). Postmating isolation occurs because hybrids are maladapted to the new environment as a result of additive genetic effects when hybrids are intermediate relative to parents, dominant genetic effects when hybrids are mismatched for different parental phenotypes (49), or epistatic genetic effects when interactions among loci create departures from additivity (50).

\section{Epistatic interactions occur in hybrids among divergent lineages}

The availability of different hybrid genomic backgrounds and detailed trait information in Florida A. sagrei allowed us to test if epistasis increases post-mating isolation among divergent 
328 clades in the native range. Under this scenario, long-term isolation of $A$. sagrei clades led to the 329 random accumulation of divergent alleles genome-wide that interact with alleles at the adaptive $330 \mathrm{X}$ chromosome locus. Thus, two native populations that are adapted to the same environment,

331 but are members of different clades, would still experience limited genetic exchange because

332 hybrids will be maladapted as a result of epistatic interactions between divergent alleles. In the

333 invasive range however, due to changes in selection pressure, hybrids are common and can

334 persist. To test for epistasis, we used genome-wide association (GWA) and included 13 traits that 335 describe the size and shape of lizards (SI Appendix, Methods and Dataset S2). Previous studies 336 have indicated that variation in all these traits might have an adaptive basis in anoles (51), 337 although most support so far has been obtained for limb length (discussed in ref. 25).

339 The linear-mixed model implemented in GEMMA (52) indicated that SNPs suggestively

340 associated with the length of the distal portion of hindlimb, including metatarsals and phalanges, 341 map to the same candidate adaptive locus that we identified on the X chromosome (Fig. 3A, B;

342 SI Appendix, Fig. S23). A stronger and genome-wide significant signal was observed for the 343 same trait at this genomic region using the asaMap association model (53), which allows effect 344 sizes to vary depending on ancestry $\left(P=3.40 \times 10^{-7}\right.$; Fig. 3A; SI Appendix, Fig. S23). As well, 345 asaMap analyses indicated that the same locus affects variation in several other components of 346 limb length (Fig. 3A; SI Appendix, Fig. S23). That the same locus is involved in the control of 347 multiple limb components is expected, given the strong positive correlation among these traits 348 across samples, after removing the effect of body size (SI Appendix, Fig. S24). Identifying the 349 genes that control variation in limb length in A. sagrei is outside the scope of this study.

350 Nonetheless, we note that among the 267 genes that span the $18 \mathrm{Mbp}$ region on the $\mathrm{X}$ 
351 chromosome, there are several candidate genes known to be involved in limb development.

352 These include Cut Like Homeobox 2 (Cux2; ref. 54), Growth Differentiation Factor 11 (GDF11;

353 ref. 55), Noggin (Nog; ref. 56), T-Box Transcription Factor 1 (Tbxl; ref. 57), and

354 Xylosyltransferase 1 (Xylt1; ref. 58). In sum, GWA findings indicate that the X chromosome

355 locus singled out by genome scan analyses affects limb length, variation of which is known to be

356 adaptive in anoles (25). These results reinforce our conclusion that positive selection (rather than

357 background selection) is acting at the $\mathrm{X}$ chromosome locus and implicate limb length as an

358 important component of adaptive divergence in the native range.

359

360 The asaMap analyses further indicated that the strength of association between alleles at the $\mathrm{X}$

361 chromosome locus and limb length phenotypes vary among the lineages that are hybridizing in

362 Florida. Specifically, for three of the four traits for which the X chromosome locus was the top

363 genome-wide association, an effect was inferred for the Western Cuba ancestry component of

364 invasive hybrids (SI Appendix, Table S2), but not for the Eastern Cuba ancestry component.

365 These results are consistent with epistatic interactions between alleles at the X chromosome limb

366 length locus and alleles of Eastern Cuba origin that are located elsewhere in the genome. While

367 epistasis has traditionally been considered in relation to intrinsic (i.e., unconditional) isolation

368 under the Bateson-Dobzhansky-Muller model (59), evidence has been accumulating for a

369 contribution of such interactions to extrinsic isolation as well (e.g., ref. 50).

371 To further investigate these results, we stratified the Florida samples based on ancestry into two

372 groups. The first of these consisted of samples with predominantly Western Cuba ancestry and

373 low heterozygosity (SI Appendix, Methods and Fig. S25). Therefore, we refer to this group as 
374 the "hybridization limited" group. The second group consisted of samples with ancestry from all

375 parental lineages and high heterozygosity (SI Appendix, Fig. S25). Therefore, we refer to these

376 samples as the "hybridization common" group. We then tested for an effect of genotype at the

377 limb length locus in each of these sample groups. In line with results presented above, when

378 considering samples with limited hybrid ancestry, we detected a large and significant effect of

379 genotype on all limb length traits but one. Effect sizes in this case ranged from moderate (PVE

$3805.7 \%-9.3 \%$ ) to large (PVE 10.03\% - 13.08\%; Fig. 3C, D). By contrast, no such effect was

381 observed in the sample group for which hybridization is common (Fig. 3C, D).

382

383 Similar patterns could arise if hybrid and non-hybrid samples differ with respect to linkage

384 between alleles at the genotyped SNP and the causal limb length SNP. This may occur in our

385 dataset, given that we used reduced representation sequencing, and therefore are likely not

386 genotyping causal variants. To evaluate this possibility, we repeated these analyses using only

387 samples with Western Cuba ancestry at the X chromosome locus (SI Appendix, Methods),

388 reasoning that linkage relationships are more likely to be similar among closely related

389 haplotypes. Results were equivalent to those for the complete dataset, as expected if epistatic

390 interactions rather than linkage disequilibrium underpin differences in effect sizes between

391 hybrid categories (SI Appendix, Fig. S26). A limitation of epistasis analyses presented above is

392 that we could not compare the effect of genotype at the limb length locus in hybrids relative to

393 decidedly non-hybrid $A$. sagrei. This limitation is because pure parental genotypes are rare in

394 Florida. Nonetheless, because of residual hybridization in the "hybridization limited" group, the

395 effect sizes that we estimate for these samples may well be conservative.

396 


\section{Phenotype-environment correlations are consistent with changes in natural}

\section{8 selection during biological invasion}

399 Previous studies of local adaptation in anoles have relied, among other methods, on phenotype-

400 environment correlations. In the native range, a positive relationship exists both interspecifically

401 and among conspecific populations between the diameter of the perches that anoles use and limb

402 length (reviewed in ref. 25). Biomechanical studies reveal the underlying basis for this

403 relationship, specifically that the optimal limb length for lizard sprint speed and agility is a

404 function of surface diameter $(60,61)$. Although this relationship has been found repeatedly in

405 natural and experimental A. sagrei populations $(25,28,29)$, it was not found in a comparison of

406 invasive A. sagrei populations in Florida (62).

407

408 To investigate whether this lack of a relationship is still the case, we used the 30 populations

409 from Florida and Southern Georgia described above for genomic and trait analyses, for which we

410 additionally obtained 1028 observations of habitat use (SI Appendix, Dataset S3). These

411 populations were chosen to avoid heavily disturbed or urban sites, such that nearly all habitat

412 measurements originated from natural vegetation, similar to Kolbe et al. (62). We found the

413 situation to be the same as in the Kolbe et al. (62) study, with no relationship between population

414 average values of relative limb length and perch diameter $\left(R^{2}=4.61 \times 10^{-5} ; P=0.97\right.$; SI

415 Appendix Fig. S27). Thus, results from phenotype-environment correlation analyses are

416 consistent with genomic results above, indicating that changes in natural selection occurred

417 during biological invasion in A. sagrei. We note, however, that a caveat of these analyses is that 418 native populations used in previous studies are predominantly from islands in the Bahamas (25, 
419 28, 29). Comparable data from Cuban populations that sourced the Florida invasion are currently 420 not available.

421

422 Conclusions

423 Our results indicate that changes in natural selection as inferred from genomic variation at a

424 large-effect adaptive X chromosome locus contribute to differences in hybridization frequency

425 among native and invasive populations of $A$. sagrei. In the native range, evidence of frequent

426 selective sweeps suggests that the $\mathrm{X}$ chromosome locus, which affects variation in limb length, is

427 an important component in the adaptive response of $A$. sagrei populations to the environment. To

428 the extent that migration occurs among contrasting environments in the native range, adaptive

429 divergence could limit gene flow among populations within or between clades. Ancestry-specific

430 association analyses, which we could perform thanks to the availability of invasive hybrids,

431 additionally showed that the same X chromosome locus is involved in epistatic interactions when

432 hybridization occurs among divergent lineages. This result indicates that native range extrinsic

433 isolation may be stronger between populations from different clades and provides an example on

434 the value of studying invasive populations for understanding evolution in the native range.

436 Although A. sagrei has been reasonably well sampled across its range in Cuba, more detailed 437 study of the contact zones between clades is needed. Combining genomic data with trait and 438 habitat data will provide in-depth information on native range gene flow and environmental 439 drivers of local adaptation. In the invasive range, natural selection as it is manifested in the 440 native range appears to have been disrupted. Here, hybrid ancestry occurs in all populations and

441 has stabilized irrespective of whether gene flow occurred within or among divergent lineages. 
443 Whether hybridization had a major role in the success of the $A$. sagrei invasion remains to be

444 definitively established. Nonetheless, available evidence points towards hybridization-mediated

445 biological invasion. Specifically, hybrid ancestry occurs in all invasive populations. Were

446 hybridization merely a consequence of repeated introductions, we would have expected to find a

447 mosaic of hybrid and non-hybrid ancestry across the invasive range. Aside from being

448 widespread, hybrid ancestry has stabilized: samples collected 15 years apart, while representing a

449 narrow snapshot in the history of invasive A. sagrei, did not reveal changes in ancestry. Thus,

450 even if currently neutral, stability of new ancestry combinations should increase the chance that

451 adaptive allele combinations are available when invasive populations are exposed to novel

452 selective pressures. 


\section{Methods}

\section{Sequencing and Variant Calling}

455 To obtain genome-wide SNP data, we used reduced representation sequencing (RADseq; SI

456 Appendix, Methods). We aligned quality-filtered reads to the $A$. sagrei reference genome v2.0

457 (63) in the dDocent v2.2.20 pipeline (64). We then performed joint genotyping using Freebayes

458 v. 1.3.2 (65) including data from the 897 A. sagrei libraries (885 samples and 12 replicates),

459 along with 128 other $A$. sagrei libraries that were part of a related project. To decrease SNP

460 calling runtime and following Freebayes manual recommendations, we only called the six best

461 alleles. We next applied stringent variant filtering and estimated post-filtering genotyping errors

462 (SI Appendix, Methods).

463

\section{Spatial population genetic structure across the range of $\boldsymbol{A}$. sagrei}

465 To summarize population structure, we used PCA in "adegenet" (v. 2.1.1; ref. 66). Fine-scale

466 population structure in Western Cuba was explored using a separate PCA. For each analysis, we

467 identified markers genotyped in at least $99 \%$ of samples with a minor allele frequency $>1 \%$.

468 From this set, to decrease computational time, we selected 10,000 random SNPs using the

469 "vcfrandomsample" tool from $v c f l i b$ (https://github.com/vcflib/vcflib). These SNPs were located 470 on chromosomes 1-5 of the v2.0 reference genome, which are equivalent to chromosomes 1-6 of

471 the v2.1 reference genome. For consistency, we will refer only to genome coordinates v2.1

472 throughout. We complemented the PCA with estimates of the A. sagrei phylogeny, Bayesian

473 clustering, identity-by-state, and isolation-by-distance (SI Appendix, Methods). 


\section{Temporal changes in the ancestry of invasive hybrids}

475 To investigate whether the ancestry of invasive hybrids has stabilized or is changing, we 476 revisited in 2018 six populations that we sampled in 2003 ( $N=172$; SI Appendix, Fig. S9). We

477 targeted the same sites, or sites located as close as possible to the original 2003 sampling. We 478 first used PCA, as described above. Additionally, we modeled temporal changes at three metrics 479 of hybrid status, as follows. First, we identified a set of ancestry informative markers (AIMs) that 480 are diagnostic of the Western Cuba lineage. To be classified as AIMs, SNPs needed to be scored 481 in $70 \%$ or more of Western Cuba and non-Western Cuba samples, and show an allele frequency 482 of $20 \%$ or lower in one group, and $80 \%$ or higher in the other. In all, 711 SNPs fit these criteria, 483 of which 469 were scored at high quality in the 2003 and 2018 invasive samples. To summarize 484 Western Cuba ancestry, we then averaged AIM allele frequency for each invasive genotype. 485

486 Second, we calculated an index of admixture $\left(H_{A}\right)$, following Keller \& Taylor (12). As input, we 487 used the STRUCTURE results from the $K=6$ analysis with prior population information (Fig. 488 1B). Third, we calculated heterozygosity using 155,905 filtered SNPs from chromosomes 1-6 489 (for details on SNP filtering, see identity-by-state analyses section, SI Appendix, Methods). To 490 test whether AIM allele frequency, $H_{A}$, or heterozygosity changed over 15 years, we used three 491 linear models in R v. 3.6.1 (67). These had each of the three metrics as the response variable, and 492 population IDs and time (2003 or 2018) as the predictor variable.

\section{The genomic signature of natural selection in $A$. sagrei}

495 We combined information from relative differentiation $\left(F_{\mathrm{ST}}\right)$, Tajima's $D$, Fay and Wu's $H$ test, 496 nucleotide diversity $(\pi)$, and absolute differentiation $\left(D_{\mathrm{xy}}\right)$. For the invasive range, we used the 
49730 populations sampled in 2018 from Florida and Southern Georgia $(N=560$; SI Appendix,

498 Dataset S1). For the native range, we used all 10 populations ( $N=134$; SI Appendix, Dataset

499 S1). Prior to performing analyses along the genome, we updated genome coordinates from v2.0,

500 which was used to align reads and call SNPs to the most recent version (v2.1), which includes

501 changes to sequence coordinates but does not differ in sequence content. We then removed

502 gametolog SNPs (i.e. SNPs resulting from Y-chromosome reads that align to the X chromosome;

503 SI Appendix, Methods), and imputed any missing data at the remaining 123,882 SNPs in

504 BEAGLE v5.0 (68).

505

506 For $F_{\mathrm{ST}}$ analyses, we used population-pairwise comparisons and calculated $F_{\mathrm{ST}}$ in non-

507 overlapping windows of $50 \mathrm{~kb}$ in VCFtools (v. 0.1.16; ref. 69). To avoid pseudoreplication, we

508 used only unique population pairs (see SI Appendix, Methods for details on population pairing).

509 We then classified windows as "outliers" if weighted $F_{\mathrm{ST}}$ was in the top $5 \%$ of observations (i.e.,

510 we sorted windowed $F_{\mathrm{ST}}$ for each population pair, and obtained windows in the top 5\%).

511 Similarly, "extreme outliers" were windows in the top 1\%. Aside from evaluating how $F_{\text {ST }}$ varies

512 along the genome, this approach additionally allowed us to investigate the repeatability of $F_{\text {ST }}$

513 differentiation, using a permutation approach implemented from Rennison et al. (ref. 70; SI

514 Appendix, Methods).

515

516 Tajima's $D$ was calculated in non-overlapping windows of 50kb in VCFtools. To estimate Fay

517 and Wu's $H$, we used "PopGenome" (v. 2.7.5; ref. 71). We first incorporated an outgroup from

518 publicly available sequence data (SI Appendix, Methods), retaining 139 SNPs scored at the

519 divergent $\mathrm{X}$ chromosome locus for all samples and for the outgroup. To get an estimate of 
520 background neutral $H$ values at the X chromosome, we obtained another set of 139 SNPs with

521 outgroup data. Similar to SNPs from the divergent X chromosome locus, these were located in

522 the male-hemizygous region, between PAR1 and PAR2. To minimize effects of genetic

523 hitchhiking which would extend the signature of selection in the vicinity of an adaptive locus,

524 candidate neutral SNPs were from the opposite end of the X chromosome (i.e., adjacent to

525 PAR1). We then compared $H$ values obtained for each range at the divergent $\mathrm{X}$ chromosome

526 locus to neutral values, and for each locus category between ranges, using Wilcoxon rank-sum

527 tests in $\mathrm{R}$, adjusting $P$ values for multiple comparisons using the Bonferroni method. Lastly,

528 while analyses of selection focused on the 2018 invasive range samples, we also contrasted $H$

529 values between the 2003 and 2018 samples, for the six invasive populations with temporal data.

530 To do this, we followed the same approach as described above for the full dataset.

531

532 To calculate nucleotide diversity $(\pi)$, and absolute differentiation $\left(D_{\mathrm{xy}}\right)$, we repeated the SNP

533 calling step for the $\mathrm{X}$ chromosome, retaining monomorphic sites as well. We filtered the output

534 keeping genotypes supported by at least four reads, and sites with data in at least $70 \%$ of samples

535 used for the genome scan analyses. Also, we removed gametolog SNPs using the same approach

536 as for the 123,882 SNP set described above, used in the rest of the genome scan analyses (see

537 also SI Appendix, Methods). We then used the python script "popgenWindows.py"

538 (https://github.com/simonhmartin/genomics_general; ref. 72) to estimate $\pi$ and $D_{\text {xy }}$ in non-

539 overlapping windows of $50 \mathrm{~kb}$ along the $\mathrm{X}$ chromosome, based on windows with at least 50 sites

540 with data. For $D_{\text {xy }}$, we used the same pairs of populations as in the $F_{\text {ST }}$ analyses. We compared

541 average per-population $\pi$ from within the divergent $X$ chromosome locus (107 windows) to a 
542 candidate neutral locus of the same size (107 windows) using the same approach as for Fay and

543 Wu's $H$ test above.

545 Genetic mapping of candidate adaptive traits in natural hybrid populations

546 We measured 13 morphological traits that describe body size (SVL), as well as the shape of

547 lizards using image analysis of X-rays (SI Appendix, Methods and Dataset S2). Measurements at

548 skeletal traits were isolated from the effect of SVL by calculating residuals from linear

549 regressions of log-transformed trait values on log-transformed SVL in R. Two GWA approaches

550 were then used: a linear mixed model in GEMMA (52), and ancestry-specific association in

551 asaMap (53). Both analyses were based on the samples obtained in 2018 from Florida and

552 Southern Georgia $(N=560)$. We filtered a VCF containing these 560 samples using the same

553 criteria as above. As well, similar to the genome scan analyses, we removed gametolog SNPs

554 and imputed any missing data that remained after filtering. For the GEMMA analyses, we used a

555 leave-one-chromosome out approach when calculating the relatedness matrix. For the asaMap

556 analyses, to account for population structure, we included as covariates the first 10 PCs from a

557 PCA constructed in "adegenet" for all samples in the analysis. Also, for both GWA approaches,

558 we included transect as an additional covariate (see SI Appendix, Methods for additional details). 


\section{Acknowledgements}

560 We thank K.A. Hodgins, L.H. Rieseberg, D.C. Card, M.D. Shapiro, and the J.B.L. and J.J.K.

561 laboratories for helpful comments, as well as J. Breeze, C. Hahn, and M. Gage for assistance

562 with lizard housing and care. This work was made possible by a Natural Sciences and

563 Engineering Research Council of Canada (NSERC) Postdoctoral Fellowship, a Banting

564 Postdoctoral Fellowship, and a Barbour award from the Harvard Museum of Comparative

565 Zoology (to D.G.B), by National Science Foundation grant DEB-1927194 (to A.J.G and J.B.L),

566 by National Science Foundation grant IOS-1827647 (to D.B.M), and by National Science

567 Foundation grant DEB-1354897 and funds from the University of Rhode Island (to J.J.K).

568 Additionally, this project was made possible through the support of a grant from the John

569 Templeton Foundation. The opinions expressed in this publication are those of the authors and 570 do not necessarily reflect the views of the John Templeton Foundation. 


\section{References}

572 1. Baker HG, Stebbins GL (1965) The Genetics of Colonizing Species (Academic, New York).

573 2. C.E. Lee, Evolutionary genetics of invasive species. Trends Ecol. Evol. 17, 386-391 (2002).

574 3. S.L. Chown, et al., Biological invasions, climate change and genomics. Evol. Appl. 8, $23-46$

575

576

577

578

579

580

581

582

583

584

585

586

587

588

589

590

591

592

593

594

595

596

597

598

599

600

601

602

603

604

605

606

607

608

609

610

611

612

613

4. D.G. Bock, et al., What we still don't know about invasion genetics. Mol. Ecol. 24, 2277-2297 (2015).

5. R.I. Colautti, J.A. Lau, Contemporary evolution during invasion: Evidence for differentiation, natural selection, and local adaptation. Mol. Ecol. 24, 1999-2017 (2015).

6. N.G. Hairston, S.P. Ellner, M.A. Geber, T. Yoshida, J.A. Fox, Rapid evolution and the convergence of ecological and evolutionary time. Ecol. Lett. 8, 1114-1127 (2005).

7. D.N. Reznick, J.B. Losos, J. Travis, From low to high gear: there has been a paradigm shift in our understanding of evolution. Ecol. Lett. 22, 233-244 (2019).

8. R.J. Abbott, Plant invasions, interspecific hybridization and the evolution of new plant taxa. Trends Ecol. Evol. 7, 401-405 (1992).

9. N.C. Ellstrand, K.A. Schierenbeck, Hybridization as a stimulus for the evolution of invasiveness in plants? Proc. Natl. Acad. Sci. USA 97, 7043-7050 (2000).

10. M. Rius, J.A. Darling, How important is intraspecific genetic admixture to the success of colonising populations? Trends Ecol. Evol. 29, 233-242 (2014).

11. J.J. Kolbe, et al., Genetic variation increases during biological invasion by a Cuban lizard. Nature 431, 177-181 (2004).

12. S.R. Keller, D.R. Taylor, Genomic admixture increases fitness during a biological invasion. J. Evol. Biol. 23, 1720-1731 (2010).

13. D.G. Bock, M.B. Kantar, C. Caseys, R. Matthey-Doret, L.H. Rieseberg, Evolution of invasiveness by genetic accommodation. Nat. Ecol. Evol. 2, 991-999 (2018).

14. H.S. Rosinger, et al., The tip of the iceberg: genome wide marker analysis reveals hidden hybridization during invasion. Mol. Ecol. 30, 810-825 (2021).

15. S.M. Hovick, K.D. Whitney, Hybridisation is associated with increased fecundity and size in invasive taxa: Meta-analytic support for the hybridisation-invasion hypothesis. Ecol. Lett. 17, 1464-1477 (2014).

16. K.D. Whitney, R.A. Randell, L.H. Rieseberg, Adaptive introgression of herbivore resistance traits in the weedy sunflower Helianthus annuus. Am. Naturalist, 167, 794-807 (2006).

17. A. Zhan, H.J. MacIsaac, M.E. Cristescu, Invasion genetics of the Ciona intestinalis species complex: From regional endemism to global homogeneity. Mol. Ecol. 19, 4678-4694 (2010).

18. E. Anderson, Hybridization of the habitat. Evolution 2, 1-9 (1948).

19. M. Todesco, et al., Hybridization and extinction. Evol. Appl. 9, 892-908 (2016).

20. B.R. Grant, P.R. Grant, Evolution of Darwin finches caused by a rare climatic event. Proc. $R$. Soc. B: Biol. Sci. 251, 111-117 (1993).

21. C.B. Heiser, Hybrid populations of Helianthus divaricatus and H. microcephalus after 22 years. Taxon 28, 71-75 (1979).

22. K.J.F. Verhoeven, et al., Population admixture, biological invasions and the balance between local adaptation and inbreeding depression. Proc. R. Soc. B: Biol. Sci. 278, 2-8 (2011). 
23. K.L. Millette, A. Gonzalez, M.E. Cristescu, Breaking ecological barriers: Anthropogenic disturbance leads to habitat transitions, hybridization, and high genetic diversity. Sci. Total Environ. 740, 140046 (2020).

24. J.J. Kolbe, A. Larson, J.B. Losos, K. de Queiroz, Admixture determines genetic diversity and population differentiation in the biological invasion of a lizard species. Biol. Lett. 4, 434437 (2008).

25. J. B. Losos, Lizards in an Evolutionary Tree: Ecology and Adaptive Radiation of Anoles (University of California Press, Berkeley, CA, 2009).

26. B.C. Lister, The nature of niche expansion in West Indian Anolis lizards I: Ecological consequences of reduced competition. Evolution 30, 659-676 (1976a).

27. B.C. Lister, The nature of niche expansion in West Indian Anolis lizards II: Evolutionary components. Evolution 30, 677-692 (1976b).

28. J.B. Losos, D.J. Irschick, T.W. Schoener, Adaptation and constraint in the evolution of specialization of Bahamian Anolis lizards. Evolution 48, 1786-1798 (1994).

29. J.B. Losos, K.I. Warheitt, T.W. Schoener, Adaptive differentiation following experimental island colonization in Anolis lizards. Nature 387, 70-73 (1997).

30. J.J. Kolbe, M. Leal, T.W. Schoener, D.A. Spiller, J.B. Losos, Founder effects persist despite adaptive differentiation: A field experiment with lizards. Science 335, 1086-1089 (2012).

31. R.M. Cox, R. Calsbeek, Survival of the fattest? Indices of body condition do not predict viability in the brown anole (Anolis sagrei). Funct. Ecol. 29, 404-413 (2015).

32. O. Lapiedra, T.W. Schoener, M. Leal, J.B. Losos, J.J. Kolbe, Predator-driven natural selection on risk-taking behavior in anole lizards. Science 360, 1017-1020 (2018).

33. R.M. Pringle, et al. Predator-induced collapse of niche structure and species coexistence. Nature 570, 58-64 (2019).

34. I.J. Wang, R.E. Glor, J.B. Losos, Quantifying the roles of ecology and geography in spatial genetic divergence. Ecol. Lett. 16, 175-182 (2013).

35. R.G. Reynolds, et al., Phylogeographic and phenotypic outcomes of brown anole colonization across the Caribbean provide insight into the beginning stages of an adaptive radiation. J. Evol. Biol. 33, 468-494 (2020).

36. P.G. Meirmans, The trouble with isolation by distance. Mol. Ecol. 21, 2839-2846 (2012).

37. R.E. Glor, et al., Partial island submergence and speciation in an adaptive radiation: A multilocus analysis of the Cuban green anoles. Proc. R. Soc. B: Biol. Sci. 271, 2257-2265 (2004).

38. A.F. Agrawal, M.C. Whitlock, Environmental duress and epistasis: How does stress affect the strength of selection on new mutations? Trends Ecol. Evol. 25,450-58 (2010).

39. J.M. Coughlan, D.R. Matute, The importance of intrinsic postzygotic barriers throughout the speciation process. Philos. Trans. R. Soc. Lond. B Biol. Sci. 375, 20190533 (2020).

40. P. Nosil, T.H. Vines, D.J. Funk, Reproductive isolation caused by natural selection against immigrants from divergent habitats. Evolution 59, 705-719 (2005).

41. A.P. Hendry, P. Nosil, L.H. Rieseberg, The speed of ecological speciation. Funct. Ecol. 21, 455-464 (2007).

42. K.A. Hodgins, D.G. Bock, L.H. Rieseberg Trait evolution in invasive species. Annu. Plant Rev. Online 1, 1-37 (2018).

43. R. Nielsen, Molecular signatures of natural selection. Annu. Rev. Genet. 39, 197-218 (2005).

44. K.E. Lohmueller, C.D. Bustamante, A.G. Clark, Detecting directional selection in the presence of recent admixture in African-Americans. Genetics 187, 823-835 (2011). 
45. P.C. Sabeti, et al., Positive natural selection in the human lineage. Science 312, 1614-1620 (2006).

46. R. Burri, Interpreting differentiation landscapes in the light of long-term linked selection. Evol. Lett. 1, 118-131 (2017).

47. D.E. Irwin, et al., A comparison of genomic islands of differentiation across three young avian species pairs. Mol. Ecol. 27, 4839-4855 (2018).

48. T.E. Cruickshank, M.W. Hahn, Reanalysis suggests that genomic islands of speciation are due to reduced diversity, not reduced gene flow. Mol. Ecol. 23, 3133-3157 (2014).

49. K.A. Thompson, M. Urquhart-Cronish, K.D. Whitney, L.H. Rieseberg, D. Schluter, Patterns, predictors, and consequences of dominance in hybrids. Am. Naturalist 197, E72-E88 (2021).

50. J.R. Dettman, C. Sirjusingh, L.M. Kohn, J.B. Anderson, Incipient speciation by divergent adaptation and antagonistic epistasis in yeast. Nature 447, 585-588 (2007).

51. L.J. Harmon, J.J. Kolbe, J.M. Cheverud, J.B. Losos, Convergence and the multidimensional niche. Evolution 59, 409-421 (2005).

52. X. Zhou, M. Stephens, Genome-wide efficient mixed-model analysis for association studies. Nat. Genet. 44, 821-824 (2012).

53. L. Skotte, E. Jørsboe, T.S. Korneliussen, I. Moltke, A. Albrechtsen, Ancestry-specific association mapping in admixed populations. Genet. Epidemiol. 43, 506-521 (2019).

54. A.T. Tavares, T. Tsukui, J.C. Izpisua Belmonte, Evidence that members of the Cut/Cux/CDP family may be involved in AER positioning and polarizing activity during chick limb development. Development 127, 5133-5144 (2000).

55. Y. Matsubara, et al., Anatomical integration of the sacral-hindlimb unit coordinated by GDF11 underlies variation in hindlimb positioning in tetrapods. Nat. Ecol. Evol. 1, 13921399 (2017).

56. K. Lehmann, et al., A new subtype of brachydactyly type B caused by point mutations in the bone morphogenetic protein antagonist NOGGIN. Am. J. Hum. Genet. 81, 388-396 (2007).

57. G. Tejedor, et al., Whole embryo culture, transcriptomics and RNA interference identify TBX1 and FGF11 as novel regulators of limb development in the mouse. Sci. Rep. 10, 3597 (2020).

58. E.K. Mis, et al., Forward genetics defines Xylt1 as a key, conserved regulator of early chondrocyte maturation and skeletal length. Dev. Biol. 385, 67-82 (2014).

59. J.A. Coyne, H.A. Orr, Speciation (Sinauer Associates, 2004).

60. J.B. Losos, B. Sinervo, The effects of morphology and perch diameter on sprint performance of Anolis lizards. J. Exp. Biol. 145, 23-30 (1989).

61. B. Vanhooydonck, A. Herrel, D.J. Irschick, Out on a limb: the differential effect of substrate diameter on acceleration capacity in Anolis lizards. J. Exp. Biol. 209, 4515-4523 (2006).

62. J.J. Kolbe, A. Larson, J.B. Losos, Differential admixture shapes morphological variation among invasive populations of the lizard Anolis sagrei. Mol. Ecol. 16, 1579-1591 (2007).

63. A.J. Geneva, et al., Chromosome-scale genome assembly of the brown anole (Anolis sagrei), a model species for evolution and ecology. (manuscript in preparation).

64. J.B. Puritz, C.M. Hollenbeck, J.R. Gold, DDOCENT: A RAD- seq, variant-calling pipeline designed for population genomics of non-model organisms. PeerJ 2, e431 (2014).

65. E. Garrison, G. Marth, Haplotype-based variant detection from short-read sequencing. arXiv preprint arXiv:1207.3907 (2012). 
66. T. Jombart, I. Ahmed, adegenet 1.3-1: new tools for the analysis of genome-wide SNP data. Bioinformatics 27, 3070-3071 (2011).

708 67. R Development Core Team. R: A Language and Environment for Statistical Computing (http://www.R-project.org/) (R Foundation for Statistical Computing, 2011).

68. B. L. Browning, S. R. Browning, Genotype imputation with millions of reference samples. Am. J. Hum. Genet. 98,116-126 (2016).

69. P. Danecek, et al., The variant call format and VCFtools. Bioinformatics 27, 2156-2158 (2011).

70. D.J. Rennison, Y.E. Stuart, D.I. Bolnick, C.L. Peichel, Ecological factors and morphological traits are associated with repeated genomic differentiation between lake and stream stickleback. Phil. Trans. R. Soc. B. 374, 20180241 (2019).

71. B. Pfeifer, U. Wittelsbürger, S.E. Ramos-Onsins, M.J. Lercher, PopGenome: An efficient Swiss army knife for population genomic analyses in R. Mol. Biol. Evol. 31, 1929-1936 (2014).

72. S.H. Martin, J.W. Davey, C.D. Jiggins, Evaluating the use of ABBA - BABA statistics to locate introgressed loci. Mol. Biol. Evol. 32, 244-257 (2014). 


\section{$722 \quad$ Figure legends}

723 Figure 1: Spatial genetic structure and diversity of $\boldsymbol{A}$. sagrei. A, PCA of all samples, and a

724 separate PCA of the Western Cuba native range subset (inset). Native genotypes are in color, 725 invasive genotypes are in grey. The arrow points to one genotype identified as a hybrid in the 726 native range. B, STRUCTURE membership, with samples grouped by range and population.

727 Each of the 44 populations is separated by white dotted lines. With the exception of one sample 728 that showed evidence of admixture, all native range genotypes are treated as learning samples (SI 729 Appendix, Methods). Cuba populations are arranged from West to East. Invasive populations are 730 arranged in decreasing order of Western Cuba ancestry. C, Heterozygosity rate and deleterious 731 SNP proportion, averaged per population. $P$ values are from two-sided Wilcoxon rank-sum tests.

Figure 2: Genome scans for selection. A, average genome-wide $F_{\mathrm{ST}}$ for the native range (top)

734 and the invasive range (bottom) for chromosomes 1-10. B, Zoomed-in view of average $F_{\text {ST }}$ along

735 the X chromosome (chromosome 7). C, Zoomed-in view of Tajima's $D$ along the X

736 chromosome, averaged for windows with Tajima's $D$ estimates at $50 \%$ or more of populations

737 within each range. For both $\mathbf{B}$ and $\mathbf{C}$, empty circles are used for the native range and filled circles

738 are used for the invasive range. Colored lines show a loess smoothing ("span" of 0.2 ) for the

739 native range (red) and the invasive range (black). The grey shading marks the $18 \mathrm{Mbp} \mathrm{X}$

740 chromosome locus where most native-range $F_{\text {ST }}$ outliers are located (SI Appendix, Fig. S12).

741 The dashed vertical lines indicate the boundaries of the pseudo-autosomal regions (PARs). D,

742 Fay and Wu's $H$, calculated within the $\mathrm{X}$ locus and at a region outside of this locus, used to

743 estimate background neutral $H$ levels. Lines connect $H$ estimates obtained for the same

744 population. $\mathbf{E}, \pi$ values for genomic windows within the divergent $\mathrm{X}$ locus and for background

745 neutral values. Grey dots show global average $\pi$. For both $\mathbf{D}$ and $\mathbf{E}, P$ values are from paired

746 Wilcoxon rank-sum tests, using averages estimated per population and locus category (Methods). 
748 Figure 3: Genetic architecture of limb length. A, Genome-wide associations for the relative

749 length of the distal portion of hindlimb, inferred using the GEMMA association model (top) and 750 the asaMap ancestry-specific association model (bottom). For the asaMap results, white dots 751 show the smallest $P$ values for three other traits for which the same locus was identified as the 752 top genome-wide association (see SI Appendix, Fig. S23 for GWA results for each trait). The red 753 dashed lines are the Bonferroni-corrected significance thresholds, while the black dashed lines 754 indicate the suggestive significance thresholds (SI Appendix, Methods). B, Overlap between 755 GWA for limb length and $F_{\mathrm{ST}}$ outliers in the native range. The upper plot indicates, for 756 chromosome 7, the position of the top 1\% SNPs identified based on strength of GWA (blue 757 lines) or $F_{\mathrm{ST}}$ (grey lines). $F_{\mathrm{ST}}$ values are from the Mariel x Guanabo population pair (SI 758 Appendix, Fig. S12). The lower plot shows asaMap association results (blue dots), and Mariel x 759 Guanabo $F_{\mathrm{ST}}$ values per SNP (grey dots) along chromosome 7. The black line is a loess 760 smoothing ("span” of 0.2) of association results. The grey shading marks the $18 \mathrm{Mbp} \mathrm{X}$ 761 chromosome divergent locus, and the dashed vertical lines indicate the boundaries of the two 762 PARs on the X chromosome. C, Relative hindlimb length for samples with small (SM) and large 763 (LG) alleles at the lead SNP identified on chromosome 7 using the asaMap model. Trait values 764 are given separately for the "hybridization limited" and the "hybridization common" sample 765 groups. Points and error bars indicate mean and standard deviation. D, Effect sizes of genotypes 766 at the same SNP as in panel C, calculated for all traits, for each of the two sample groups 767 separately. Asterisks indicate significant main effects of genotype after Bonferroni correction. 768 For each sample group ancestry proportions are shown, as estimated using a STRUCTURE 769 analysis at $K=2$ and $K=6$ (see also SI Appendix, Fig. S25). 